\title{
Sulfonic acid functionalised SBA-15 as catalysts for Beckmann rearrangement and esterification reaction
}

\author{
Wei Zhao · Petra Salame · Franck Launay • \\ Antoine Gédéon · Zhengping Hao
}

Published online: 30 May 2007

(C) Springer Science+Business Media, LLC 2007

\begin{abstract}
Hybrid inorganic-organic solid acid materials SBA-15-Ph- $\mathrm{SO}_{3} \mathrm{H}$ were synthesized by the directly cocondensation method under the acid medium. The resulting materials perform well both in the liquid phase Beckmann rearrangement (The yield is 32.3\%) and esterification reaction (The yield is $95.5 \%$ ). And more, the catalysts show the different catalytic performance in these two reactions.
\end{abstract}

Keywords Beckmann rearrangement - Esterification reaction $\cdot$ SBA-15 - Arenesulfonic acids

\section{Introduction}

At present, the more and more harsh environmental rules incite the companies/factories to improve their technology vigorously and make the manufacture procedure more environment compatible. The conversion of cyclohexanone oximes into the corresponding lactams that is the raw material of Nylon 6 is one of the most important industrial reactions [1]. Conventionally, this process employs the fuming sulfuric acid as a catalyst [2] due to the reasons that this reaction only requires the catalytic amount of a strong acid and that the fuming sulfuric acid can prevents the secondary reactions such as hydrolysis. However, the using

\footnotetext{
W. Zhao $(\bowtie) \cdot$ Z. Hao

Research Center for Eco-Environmental Sciences, Chinese

Academy of Sciences, Beijing 100085, P. R. China

e-mail: zhaowei@mail.rcees.ac.cn

P. Salame · F. Launay · A. Gédéon $(\bowtie)$

Laboratoire SIEN-CNRS, (UMR 7142), Université P. e t M.

Curie, 4 place Jussieu, 75252 Paris, France

e-mail: ag@ccr.jussieu.fr
}

of fuming surfuric acid brings about a number of disadvantages like the large amount of low-value by-product (ammonium sulphate), the inherent corrosive nature of the acid and violent exothermic reaction. In order to overcome these inconveniences, a wide variety of solid acid was studied to instead of the fuming surfuric acid. Based on the different vapour-phase Beckmann rearrangement systems, all sorts of the acidity, such as, the strong [3], the medium [4], the weak acid [5] and even the neural silanol [6], were reported that are responsible for this rearrangement procedure. On the contrary, only the strong Brönster acid site [7-8] shows a good performance for this conversion in the liquid-phase Beckmann rearrangement. Ethyl caprylate (EP) is widely used in the manufacturing of cosmetics or in food industry [9] and large-scale preparation of this product also need the liquid acid as catalysts. Since ethyl caprylate is related to the human consumption, the product has to be as pure as possible, and, thus, trace impurities from corrosion products are not allowed. In this paper, we synthesize the arenesulfonic functionalized SBA-15 via direct one-step synthesis method. The obtained SBA-15$\mathrm{Ph}-\mathrm{SO}_{3} \mathrm{H}$ materials show a good catalytic performance on the typical strong acid catalysis reactions. Interesting, the $-\mathrm{Ph}-\mathrm{SO}_{3} \mathrm{H}$ groups play a different role in the liquid phase Beckmann rearrangement of cyclohexanone oxime (CHO) and esterification of caprylic acid (CA).

\section{Experimental}

Arenesulfonic functionalized mesoporous materials were synthesized as follows: $4 \mathrm{~g}$ Pluronic 123 were dissolved in $125 \mathrm{ml}$ of $2 \mathrm{M} \mathrm{HCl}$ at room temperature. The solution was heated to $40{ }^{\circ} \mathrm{C}$ before adding TEOS. After $45 \mathrm{~min}$ of 
TEOS prehydrolysis, 2-(4-chlorosulfonylphenyl) ethyltrimethoxysilane (CSPTMS) was added to the mixture. The molar composition of the resulting solution for $4 \mathrm{~g}$ of copolymer was $1+x \mathrm{SiO}_{2}: x$ CSPTMS: $6.5 \mathrm{HCl}: 180 \mathrm{H}_{2} \mathrm{O}$ with $x=0.05,0.1,0.15$ and 0.2 , respectively. The mixture was stirred for $24 \mathrm{~h}$ at $40{ }^{\circ} \mathrm{C}$ and then aged at $100{ }^{\circ} \mathrm{C}$ for $24 \mathrm{~h}$ under static conditions. The solid product was recovered by filtration and air-dried at room temperature overnight. The template was first removed by extraction with ethanol under reflux for $24 \mathrm{~h}$, then by calcination in air at $200{ }^{\circ} \mathrm{C}$ for $24 \mathrm{~h}$. The X-ray powder diffraction (XRD) data were acquired on a Bruker-axs D8 device using $\mathrm{Cu} \mathrm{k} \alpha$ radiation. The data were collected from 0.6 to $5^{\circ}$ with a resolution of $0.02^{\circ}$. Nitrogen adsorption and desorption isotherms at $77 \mathrm{~K}$ were measured using Micromeritics ASAP 2010 system. The data were analyzed using the BJH model and the pore size distributions were calculated by the desorption branches. Sulfur and silicon composition of SBA-15-Ph- $\mathrm{SO}_{3} \mathrm{H}$ samples were determined by elemental analysis in CNRS Analysis center in Vernaison (France). The acid number of SBA-15- $\mathrm{SO}_{3} \mathrm{H}$ samples were measured by the in situ thermal desorption experiment which carried out by treatment of the samples at increase temperature in vacuum $1 \mathrm{~h}$ each time. The catalysis tests were carried out in a $50 \mathrm{ml}$ two-necked flask equipped with a reflux condenser. The products were analyzed by gas chromatography (GC) using a Delsi-Nermag 200 instrument and a $25 \mathrm{~m}$ capillary column of cross linked 5\% phenylmethylsilicone.

\section{Results and discussion}

The results of $\mathrm{N}_{2}$ adsorption-desorption isotherms measurements for the different SBA-15 samples prepared with various concentrations of CSPTMS in the initial mixture (Fig. 1) suggest that all the samples are the mesoporous materials.

Among them, the isotherm of sample $b$ and $c$ are the typical type IV isotherm, like that of pure SBA-15 (sample a). However, the isotherms of sample $d$ and e that have the CSPTMS/(CSPTMS + TEOS) ratio above 0.1 in the initial mixture are obviously different from the type IV isotherm. Indeed, there are two hysteresis loops as a result of a double pore diameter distribution appear in these isotherms. There is another smaller pore about $39 \AA$ besides the $60 \AA$ one which present solely in the pure silica SBA15 material, obviously exist in these two samples. And for sample e, this pore $(39 \AA)$ became the major one (The volume of this pore is $74 \%$ of the total pore volume). In order to know the reason and the stability for this two pores system, we calcined the sample e under a drastic condition $\left(600{ }^{\circ} \mathrm{C}\right)$. The resulting sample (Fig. 1f) shows the two
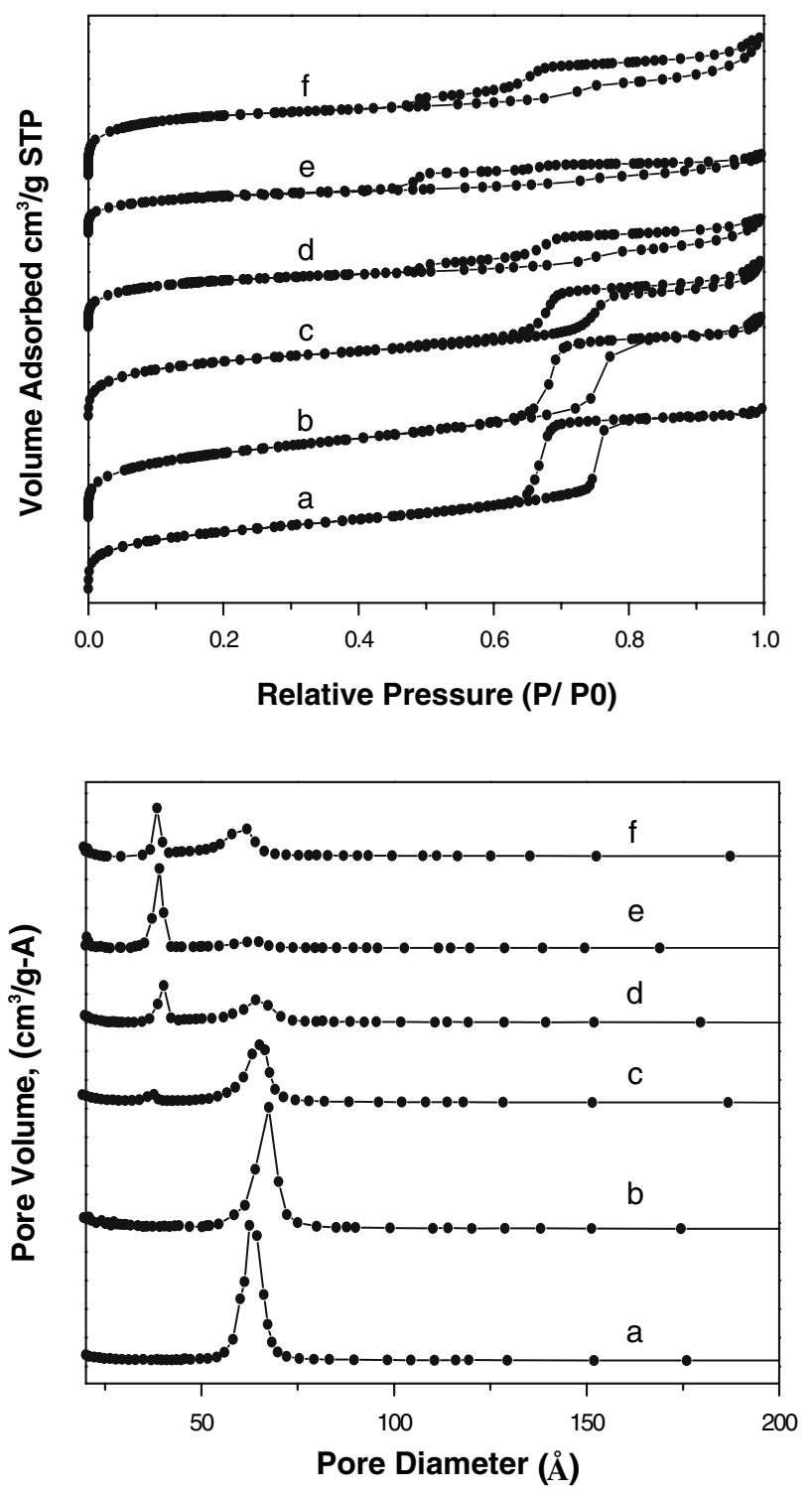

Fig. $1 \mathrm{~N}_{2}$ adsorption/desorption isotherm (left) and pore size distributions (right) of SBA-15- $\mathrm{SO}_{3} \mathrm{H}$ samples. a: Pure-SBA-15; b: $\mathrm{CSPTMS} / \mathrm{SiO}_{2}+\mathrm{CSPTMS}=0.05 ; \mathrm{c}: \mathrm{CSPTM} \mathrm{S}$ $\mathrm{SiO}_{2}+\mathrm{CSPTMS}=0.1 ;$ d: $\mathrm{CSPTMS} / \mathrm{SiO}_{2}+\mathrm{CSPTMS}=0.15 ;$ e: $\mathrm{CSPTMS} / \mathrm{SiO}_{2}+\mathrm{CSPTMS}=0.2$; f: Sample e calcined at $600{ }^{\circ} \mathrm{C}$

pores system is still exist while the part of the big pore $(60 \AA)$ become the major pore again (The volume of this pore is $77 \%$ of the total pore volume, while, it is only $26 \%$ for sample e). This result is corresponded well to the results of XRD. The XRD pattern of sample e (Fig. 2) is improved greatly and corresponds to the typical hexagonal texture structure after calcinations at $600{ }^{\circ} \mathrm{C}$ (Fig. 2f).

Based on above results, we think the two pores system mainly forms at the self-assembling process while the introduction of arenesulfuric group into the channel of SBA-15 also can cause partly the two pores system. Both the reason that the two pores system loses the order of the hexagonal pore structure and the reason that the introduction 


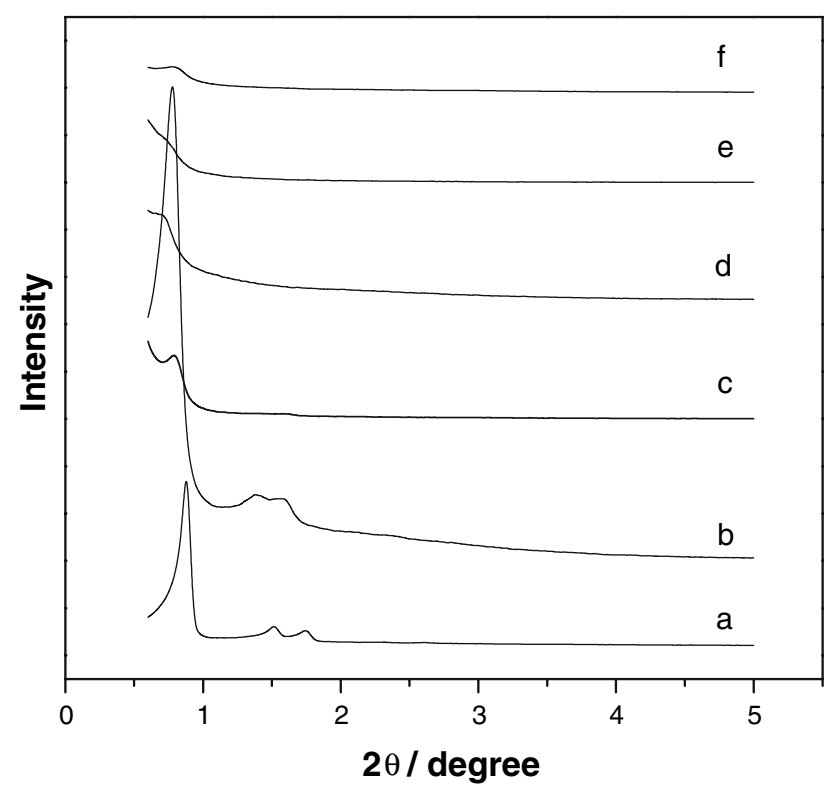

Fig. 2 XRD patterns of of SBA-15- $\mathrm{SO}_{3} \mathrm{H}$ samples. a: Pure-SBA-15; b: CSPTMS $/ \mathrm{SiO}_{2}+\mathrm{CSPTMS}=0.05 ; \mathrm{c}:$ CSPTMS $/$ $\mathrm{SiO}_{2}+\mathrm{CSPTMS}=0.1 ;$ d: $\mathrm{CSPTMS} / \mathrm{SiO}_{2}+\mathrm{CSPTMS}=0.15 ;$ e: $\mathrm{CSPTMS} / \mathrm{SiO}_{2}+\mathrm{CSPTMS}=0.2$; f: Sample e calcined at $600{ }^{\circ} \mathrm{C}$

of arenesulfuric group into the channel of SBA-15 can lead to a substantial loss in scattering contrast between the channel and the wall [10] are responded to the poor XRD pattern of these functionalized materials. Simultaneously, the two pore systems also suggest that the arenesulfuric group probably concentration in some part of the channel in contrast to distribute orderly in the channel. That's why the big pore $(60 \AA)$ can be recovered obviously after calcinations of $600{ }^{\circ} \mathrm{C}$. The sulfur amount and acid number increase lineally with the amount of CSPTMS in initial mixture (Table 1) also suggests that the arenesulfonic groups had been incorporated into the channel of SBA-15 successfully.

Just as shown in Scheme 1, since the part of 2(4-chlorosulfonylphenyl) ethyl $\left(-\mathrm{CH}_{2} \mathrm{CH}_{2}-\mathrm{Ph}-\mathrm{SO}_{2} \mathrm{Cl}\right)$ contains the more hydrophobic organic groups of ethyl and benzene ring (with $\pi$ electron) that has powerful dispersion force with the Pluronic 123, so, this part will prefer to enter the organic phase (P123) during the self-assemble procedure. It means the arenesulfonic groups will be only inside of mesoporous walls but no other choice, such as, be into or on the surface of mesoporous walls.

We first tested the catalytic properties of the new materials in the liquid phase Beckmann rearrangement of cyclohexanone oxime to produce $\varepsilon$-caprolactam $(\mathrm{CL})$. Learning from the results of Table 2, we found: (1) for the pure SBA-15 sample, there is nearly no conversion of oxim.

(2) for the sulfonic acid functionalised SBA-15, the acid number of the catalyst has a good line relationship with the selectivity of $\mathrm{CL}$ while the conversion of $\mathrm{CHO}$ also increase slightly with the enhance of acid number, but it begin to decrease for sample e (Fig. 3). The first result suggests that the cyclohexanone oxime cannot be absorbed
Table 1 The results of elemental analysis for different SBA-15- $\mathrm{SO}_{3} \mathrm{H}$ materials

\begin{tabular}{llll}
\hline Material & $\mathrm{S}(\mathrm{wt} \%)$ & $\mathrm{Si}(\mathrm{wt} \%)$ & Acid number(mmol $/ \mathrm{g})$ \\
\hline $\mathrm{CSPTMS} / \mathrm{SiO}_{2}+\mathrm{CSPTMS}=0.05$ & 2.28 & 34.26 & 0.61 \\
$\mathrm{CSPTMS} / \mathrm{SiO}_{2}+\mathrm{CSPTMS}=0.1$ & 3.66 & 30.68 & 1.14 \\
$\mathrm{CSPTMS} / \mathrm{SiO}_{2}+\mathrm{CSPTMS}=0.15$ & 4.37 & 29.74 & 1.37 \\
$\mathrm{CSPTMS} / \mathrm{SiO}_{2}+\mathrm{CSPTMS}=0.2$ & 5.31 & 27.65 & 1.66 \\
\hline
\end{tabular}

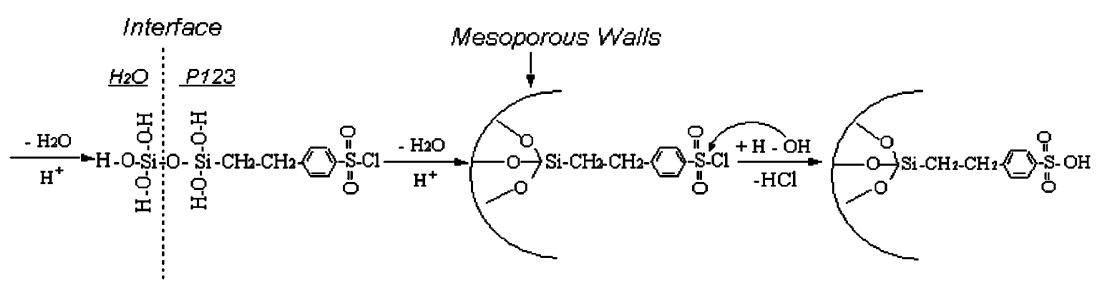

\section{Scheme 1}


Table 2 Catalytic activities of different SBA-15- $\mathrm{SO}_{3} \mathrm{H}$ materials (1)

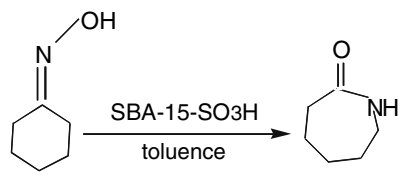

\begin{tabular}{|c|c|c|c|c|c|}
\hline Material & Acid number $(\mathrm{mmol} / \mathrm{g})$ & Conv. of $\mathrm{CHO}(\%)$ & Selec. of CL (\%) & Yield $(\%)$ & $\mathrm{TON}^{\mathrm{a}}$ \\
\hline No & 0 & 0 & - & 0 & - \\
\hline Pure-SBA-15 & 0 & 1.7 & 0 & 0 & - \\
\hline $\mathrm{CSPTMS} / \mathrm{SiO}_{2}+\mathrm{CSPTMS}=0.05$ & 0.61 & 47.0 & 20.7 & 9.7 & 1.47 \\
\hline $\mathrm{CSPTMS} / \mathrm{SiO}_{2}+\mathrm{CSPTMS}=0.1$ & 1.14 & 52.3 & 41.6 & 21.8 & 1.69 \\
\hline $\mathrm{CSPTMS} / \mathrm{SiO}_{2}+\mathrm{CSPTMS}=0.15$ & 1.37 & 53.0 & 51.5 & 27.3 & 1.76 \\
\hline $\mathrm{CSPTMS} / \mathrm{SiO}_{2}+\mathrm{CSPTMS}=0.2$ & 1.66 & 49.6 & 66.5 & 33.0 & 1.76 \\
\hline
\end{tabular}

Reaction conditions: catalyst: $0.1 \mathrm{~g}$; solvent: $20 \mathrm{ml}$ (toluene); cyclohexanone oxime $(\mathrm{CHO}): 0.1 \mathrm{~g} ; 130{ }^{\circ} \mathrm{C} ; 24 \mathrm{~h}$

a TON: $\varepsilon$-caprolactam(mol)/Acid(mol)

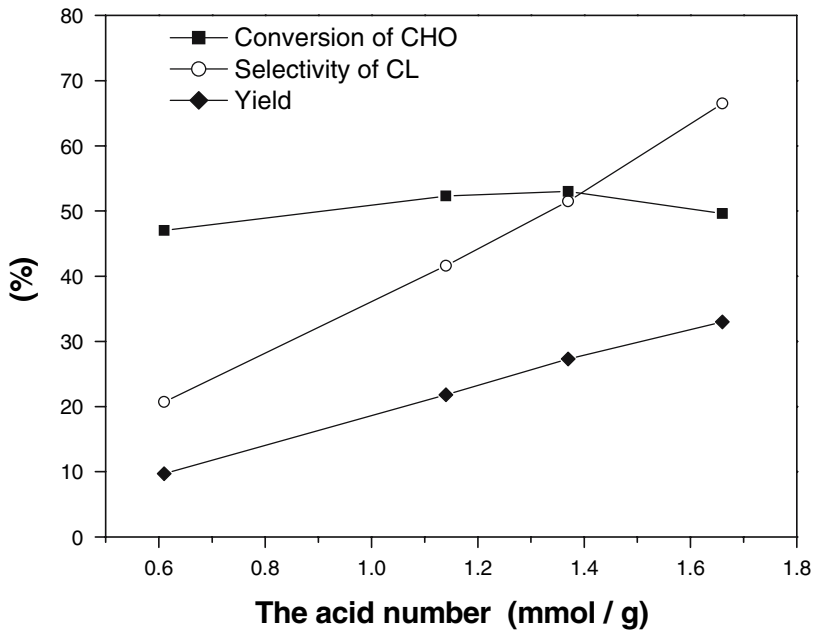

Fig. 3 The relationship between the catalytic activity and the acid number of catalysts by the surface of pure-SBA-15 easily and/or the neural silanol couldn't catalyze this reaction.

Based on the second result, we think the value of TON probably can be seemed as a constant (5/3) in this reaction. It's means this reaction system maybe needs the chemical amount strong acid as catalyst and per three acid sites can transform five substrate molecules to product. The part of narrow channel can plays as a "shape selectivity" role for this reaction. Both the electric and the geometric arrangement of this part are advantage to the transform of $\mathrm{CHO}$ to CL. So, the more the sulfonic acid group exist in the channel, the higher the selectivity of CL will be obtained. Since the $39 \AA$ narrow pore becomes the major pore for sample e, so the diffusion speed of substrate and products in this sample will be slow. As a result, the conversion of $\mathrm{CHO}$ begins to decrease.

Table 3 Catalytic activity of different SBA-15- $\mathrm{SO}_{3} \mathrm{H}$ materials (2)

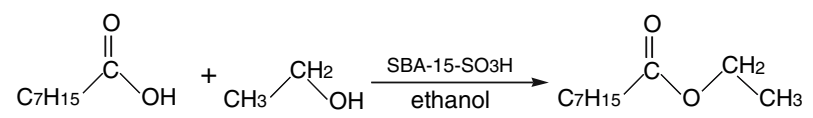

\begin{tabular}{|c|c|c|c|c|c|}
\hline Materials & Acid number $(\mathrm{mmol} / \mathrm{g})$ & Conv. of CA $(\%)$ & Selec. of EP $(\%)$ & Yield $(\%)$ & $\mathrm{TON}^{\mathrm{a}}$ \\
\hline No & - & 3.1 & 100 & 3.1 & - \\
\hline Pure-SBA-15 & 0 & 3.8 & 100 & 3.8 & - \\
\hline $\mathrm{CSPTMS} / \mathrm{SiO}_{2}+\mathrm{CSPTMS}=0.05$ & 0.61 & 95.5 & 100 & 95.5 & 109.6 \\
\hline $\mathrm{CSPTMS} / \mathrm{SiO}_{2}+\mathrm{CSPTMS}=0.1$ & 1.14 & 92.1 & 100 & 92.1 & 56.6 \\
\hline $\mathrm{CSPTMS} / \mathrm{SiO}_{2}+\mathrm{CSPTMS}=0.15$ & 1.37 & 87.9 & 100 & 87.9 & 44.9 \\
\hline $\mathrm{CSPTMS} / \mathrm{SiO}_{2}+\mathrm{CSPTMS}=0.2$ & 1.66 & 80.1 & 100 & 80.1 & 33.8 \\
\hline
\end{tabular}

Reaction conditions: catalyst: $0.1 \mathrm{~g}$; solvent: $25 \mathrm{ml}$ (ethanol); $n$-caprylic acid $(\mathrm{CA}): 7 \mathrm{mmol} ; 80^{\circ} \mathrm{C} ; 5 \mathrm{~h}$

a TON: Ethyl caprylate (mol)/Acid(mol) 
Another application of the new materials was studied in the esterification of Ethyl caprylate (EP). On the contrary to the above experiment results, the sample with less arenesulfonic groups but better channel structure showed the highest conversation meanwhile the selectivity of EP are similar for all samples. And more, only the catalytic amount of strong acid is necessary for this reaction (Table 3). In this case, we think the procedure of this reaction is more simple and it is not necessary provide a special environment for the forming of the product.

The more the channel is advantage to the diffusion of substrate and products the faster the reaction can be proceed.

\section{Conclusions}

In conclusion, the arenesulfonic groups had been incorporated into the channel of SBA-15 successfully and probably concentration in some part of the channel. Based on the different reaction mechanism, the chemical amount strong acid and the catalytic amount strong acids are necessary for the liquid phase beckmann rearrangement and esterification reaction respectively. We believe these new type solid acid materials will have wide application in the chemical industry.

Acknowledgements Thanks for the financial support from the National Basic Research Program of China (2004CB719504) and the key project of the knowledge innovation of China Academy of Sciences (KICX3-SW-430).

\section{References}

1. M.A. Camblor, A. Corma, H. Garcia, V.S. Herledan, S. Valencia, J. Catal. 177, 267 (1998)

2. J.E. Kent, Riegel's Handbook of Industrial Chemistry, 8th edn. (Van Nostrand, New York, 1983), p. 402

3. A. Aucejo, M.C. Barguet, A. Corma, V. Fornes, Appl. Catal. A: Gen 22, 187 (1986)

4. L.X. Dai, R. Hayasaka, Y. Iwaki, K.A. Koyano, T. Tasumi, Chem. Commun. 1071 (1996)

5. T. Curtin, J.P. Mcmonagle, B.K. Hoodnett, Appl. Catal. A: Gen 93, 917 (1992)

6. H. Sato, K. Hirose, M. Kitamura, Y. Nakamura, Stud. Surf. Sci. Catal. 49, 1213 (1989)

7. A. Corma, H. Garcia, J. Primo, J. Catal. 11, 593 (1991)

8. Y.M. Chung, H.K. Rhee, J. Mol. Cat. A 159, 389 (2000)

9. W.H. Hwu, J.S. Cheng, K.W. Cheng, Y.P. Chen, J. Supercrit. Fluid. 28, 1 (2004)

10. I. Kinski, H. Gies, F. Marlow, Zeolite 19, 375 (1997) 